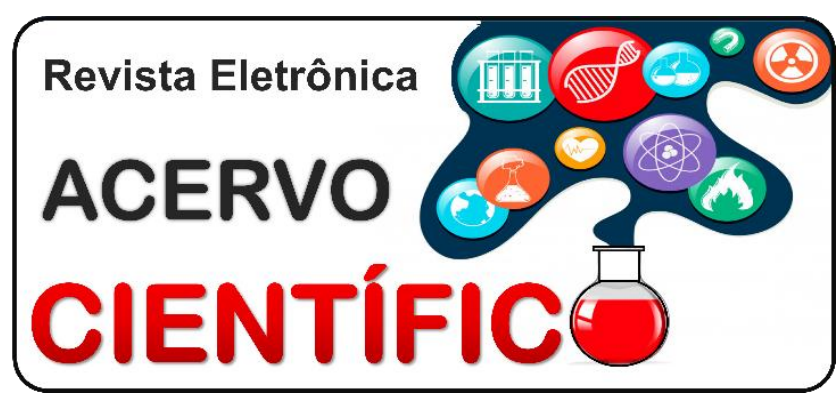

\section{ARTIGO ORIGINAL}

Recebido em: 4/2020

Aceito em: 4/2020

Publicado em: $6 / 2020$

\title{
As diversidades da predominância da sífilis adquirida nas regiões do brasil (2010 - junho de 2019)
}

Diversities of the predominance of syphilis acquired in brazilian regions (2010 - june 2019)

Diversidades de la predominancia de sífilis adquiridas en las regiones brasileñas (2010 junio 2019)

Letícia Goes Santos ${ }^{1 *}$, Amanda Silveira Carvalho Dantas ${ }^{1}$, Lucas Ferreira de Sá Santos ${ }^{1}$, Izailza Matos Dantas Lopes ${ }^{1}$, Rute de Oliveira Farias ${ }^{1}$, Maria Natália da Silva Montalvão ${ }^{1}$, Catarine Cruz Matos $^{1}$, Rodrigo Ribeiro de Almeida ${ }^{1}$, Victória Silveira Abril ${ }^{1}$, Osmar Ribeiro de Jesus Neto ${ }^{1}$.

Resumo: Esse artigo buscou avaliar a prevalência de Sífilis Adquirida em território brasileiro no período de 2010 a junho de 2019. Foi realizado um estudo transversal, observacional, retrospectivo e de caráter analítico e quantitativo, a partir de uma coleta de dados do Sistema de Informação de Agravos de Notificação (SINAN) do Ministério da Saúde no período de 2010 a junho de 2019. Foi observado um aumento dos casos de Sífilis Adquirida no Brasil, com maior taxa de incidência na região Sudeste e a menor na região Norte. Percebeu-se que o perfil das pessoas acometidas por Sífilis Adquirida, no Brasil, no mesmo intervalo de tempo, foi do gênero masculino, com 59,6\%; com idade entre 20 a 29 anos, 33,6\%; analfabetos $(1,2 \%)$, com ensino fundamental incompleto ( $1^{\mathrm{a}}$ a $4^{\mathrm{a}}$ incompleta $(5,8 \%)$, $4^{\mathrm{a}}$ completa $(3,6 \%)$ e $5^{\mathrm{a}}$ a $8^{\mathrm{a}}$ incompleta $(12,1 \%)$ e completo $(7,9 \%)$; e de cor branca $(37,8 \%)$, parda $(34,2 \%)$ e preta $(9,7 \%)$. O estudo das taxas de Sífilis Adquirida é uma ferramenta de grande valia para a saúde pública, possibilitando o planejamento de prevenção e controle de acordo com a epidemiologia, estimulando-se à adesão ao tratamento e seu seguimento.

Palavras-chave: Sífilis adquirida, Transmissão, Vigilância epidemiológica.

Abstract: This article sought to assess the prevalence of Syphilis Acquired in Brazilian territory from 2010 to June 2019. A cross-sectional, observational, retrospective and analytical and quantitative study was carried out, based on data collection from the Health Information System of Notification (SINAN) of the Ministry of Health from 2010 to June 2019. There was an increase in cases of Acquired Syphilis in Brazil, with the highest incidence rate in the Southeast and the lowest in the North. It was noticed that the profile of people affected by Acquired Syphilis, in Brazil, in the same time interval, was male, with 59.6\%; aged 20 to 29 years, 33.6\%; illiterate (1.2\%), with incomplete primary education (1st to 4 th incomplete $(5.8 \%)$, 4th complete $(3.6 \%)$ and 5 th to 8 th incomplete $(12.1 \%))$ and complete $(7.9 \%)$; and white $(37.8 \%)$, brown $(34.2 \%)$ and

1 Universidade Tiradentes (UNIT), Aracaju - Sergipe. *E-mail: leticia.gsantos1997@gmail.com 
black (9.7\%). The study of acquired syphilis rates is a valuable tool for public health, enabling prevention and control planning according to epidemiology, encouraging adherence to treatment and its follow-up.

Keywords: Acquired syphilis, Streaming, Epidemiological surveillance.

Resumen: Este artículo buscaba evaluar la prevalencia de sífilis adquirida en el territorio brasileño desde 2010 hasta junio de 2019. Se realizó un estudio transversal, observacional, retrospectivo y analítico y cuantitativo, basado en la recopilación de datos del Sistema de Información de Salud. De Notificación (SINAN) del Ministerio de Salud de 2010 a junio de 2019. Hubo un aumento en los casos de sífilis adquirida en Brasil, con la tasa de incidencia más alta en el sureste y la más baja en el norte. Se observó que el perfil de las personas afectadas por Sífilis Adquirida, en Brasil, en el mismo intervalo de tiempo, era masculino, con 59.6\%; de 20 a 29 años, 33,6\%; analfabetos (1,2\%), con educación primaria incompleta (1ํ a $4^{\circ}$ incompleto $(5,8 \%)$, 4o completo $(3,6 \%)$ y $5^{\circ}$ a $8^{\circ}$ incompleto $(12,1 \%)$ y completo $(7,9 \%)$; y blanco $(37.8 \%)$, marrón (34.2\%) y negro (9.7\%). El estudio de las tasas de sífilis adquirida es una herramienta valiosa para la salud pública, ya que permite la planificación de la prevención y el control según la epidemiología, fomentando la adherencia al tratamiento y su seguimiento.

Palabras clave: Sífilis adquirida, Transmisión, Vigilancia epidemiológica.

\section{INTRODUÇÃO}

A sífilis é uma doença infectocontagiosa e sexualmente transmissível, que tem como agente etiológico o Treponema pallidum. Possui um quadro clínico característico, dividido por fases. A Sífilis Primária é caracterizada pelo aparecimento do cancro duro. $\mathrm{Na}$ Secundária, há lesões mucocutâneas papuloescamosas e pustulosas. A Latente é assintomática e pode ser precoce (1ํano) ou tardia (acima de 1 ano ou de duração desconhecida). Já a Terciária dá início a lesões diversas, como ósseas, neurológicas e cardiovasculares (KASPER DL e FAUCI AS, 2015; VERONESI R e FOCACCIA R, 2005).

O medicamento de escolha para o tratamento da sífilis é a Benzilpenicilina Benzatina, tendo como via preferencial a região ventro-glútea, mas existem outras opções de acordo com a fase em que o paciente se encontra. Na sífilis primária, secundária e latente recente, prefere-se a Benzilpenicilina benzatina 2,4 milhões UI, IM, dose única (1,2 milhão UI em cada glúteo), e como opção a Doxiciclina 100mg, 12/12h, VO, por 15 dias. Na sífilis latente tardia ou latente com duração ignorada e sífilis terciária, faz-se uso de Benzilpenicilina benzatina 1x/semana por 3 semanas (dose total: 7,2 milhões UI), e também como opção a Doxiciclina, mas por 30 dias. A partir do correto tratamento, obtém-se bom prognóstico (BRASIL, 2019).

O Ministério da Saúde elaborou a Agenda de Ações Estratégicas para Redução da sífilis no Brasil, que tem como objetivos ampliar a cobertura do diagnóstico e tratamento; incentivar os profissionais de saúde a administrar penicilina benzatina na Atenção Básica; qualificar informações epidemiológicas, notificação e investigação; fortalecer as ações de saúde sexual e reprodutiva, especialmente no âmbito da Atenção Básica, dentre outros (BRASIL, 2017).

Apesar dos projetos para diminuição da sífilis no Brasil, observou-se aumento, nas últimas décadas, de notificações de casos de sífilis a partir do aprimoramento do sistema de vigilância e da ampliação da realização de testes rápidos. A sua taxa de detecção foi de 2,0 casos por 100 mil habitantes em 2010 a 34,1 casos por 100 mil habitantes em 2015. E em 2018, foram notificados, no SINAN, 158.051 casos (75,8 casos/100 mil habitantes) (BRASIL, 2019).

A sífilis adquirida tornou-se uma doença de notificação compulsória através da Portaria ํㅜ 2.472 em 31 de agosto de 2010, o que permitiu a real análise da epidemiologia da doença em nosso território e, portanto, o aumento da preocupação em tratar os doentes (BRASIL, 2019).

Diante do exposto, objetivou-se realizar um estudo sobre a sífilis adquirida e sua incidência no território brasileiro e em suas regiões, tendo em vista o aumento dos seus casos com o decorrer dos anos. 


\section{MÉTODOS}

O presente estudo é transversal, observacional, retrospectivo e de caráter analítico e quantitativo, que tem como instrumento de coleta de dados o Sistema de Informação de Agravos de Notificação (SINAN) do Ministério da Saúde. As informações incluídas na pesquisa são referentes aos casos notificados e confirmados de sífilis adquirida desse sistema no período de 2010 a 30 de junho de 2019.

Efetuou-se o estudo da visão epidemiológica da sífilis adquirida de todas as regiões do País, e as variáveis utilizadas foram retiradas das fichas de investigação do SINAN da Sífilis Adquirida. Comparou-se a prevalência da doença de acordo com as regiões do País, a escolaridade, o gênero, a faixa etária e a raça/cor das pessoas acometidas. Foram realizadas, ainda, pesquisas e leituras de diversos artigos em sites de pesquisas como: Scielo (Scientific Electronic Library Online) e PubMed (Publicações Médicas), restringindo-se aos achados com assuntos relacionados ao tema e com data de publicação de 2015 a 2019.

O conteúdo do presente estudo é apenas documental, através de prontuário eletrônica disponível no DATASUS (Departamento de Informática do SUS) dessa forma, são liberados o TCLE (Termo de Consentimento Livre e Esclarecido) e o TALE (Termo de Assentimento Livre e Esclarecido), como também a submissão na plataforma Brasil, devido a inexistência de contato direto com os pacientes envolvidos nos dados do SINAN, com também a disponibilidade dos mesmos em uma plataforma acessível a todos.

Após a avaliação dos citados dados do SINAN, os mesmos foram inseridos em respectivas tabelas e planilhas do programa Excel, onde foram realizadas análises descritivas. A análise estatística utilizada foi a descritiva e inferencial. As variáveis categóricas foram apresentadas por meio de frequências simples e relativas, que caracteriza a análise descritiva. Na parte inferencial, foi utilizado o teste qui-quadrado, para encontrar associação entre as variáveis. O nível de significância estatística estipulado foi de $5 \%(p \leq 0,05)$ e todos os testes foram bicaudais. O software utilizado para as análises foi o Statistical Package for the Social Sciences (IBM SPSS 25.0).

\section{RESULTADOS}

Ao decurso do período de realização das análises, observou-se aumento dos casos de Sífilis Adquirida com maior taxa de incidência na região Sudeste e a menor na região Norte. Percebeu-se que o perfil das pessoas acometidas por Sífilis Adquirida, no Brasil, no mesmo intervalo de tempo, foi do gênero masculino $59,6 \%(n=387.312)$; com idade entre 20 a 29 anos, 33,6\% $(n=218.405)$; com ensino médio completo, $17,0 \%(n=110.295)$; e de cor branca, 37,8\% $(n=245.827)$.

No Brasil, quanto à distribuição dos casos notificados, a taxa de detecção, por 100.000 habitantes, de sífilis adquirida em 2010 foi de $2,1 \%$ ( $n=3.929)$; em 2011, 9,5\% ( $n=18.317)$; em 2012, 14,5\% ( $n=28.082)$; em 2013, 19,7\% ( $n=39.566)$; em 2014, 25,1\% ( $n=50.876)$; em 2015, 34,1\% ( $n=69.729)$; em 2016, 44,5\% ( $n=91.728)$; em 2017, 59,1\% ( $n=122.679)$; em 2018, 75,8\% ( $n=158.051)$; e, no primeiro semestre de 2019 , teve um total de 67.301 casos (Figura 1). 
Figura 1 - Número de casos de Sífilis Adquirida no Brasil nos anos de 2010 a 30 de junho de 2019.

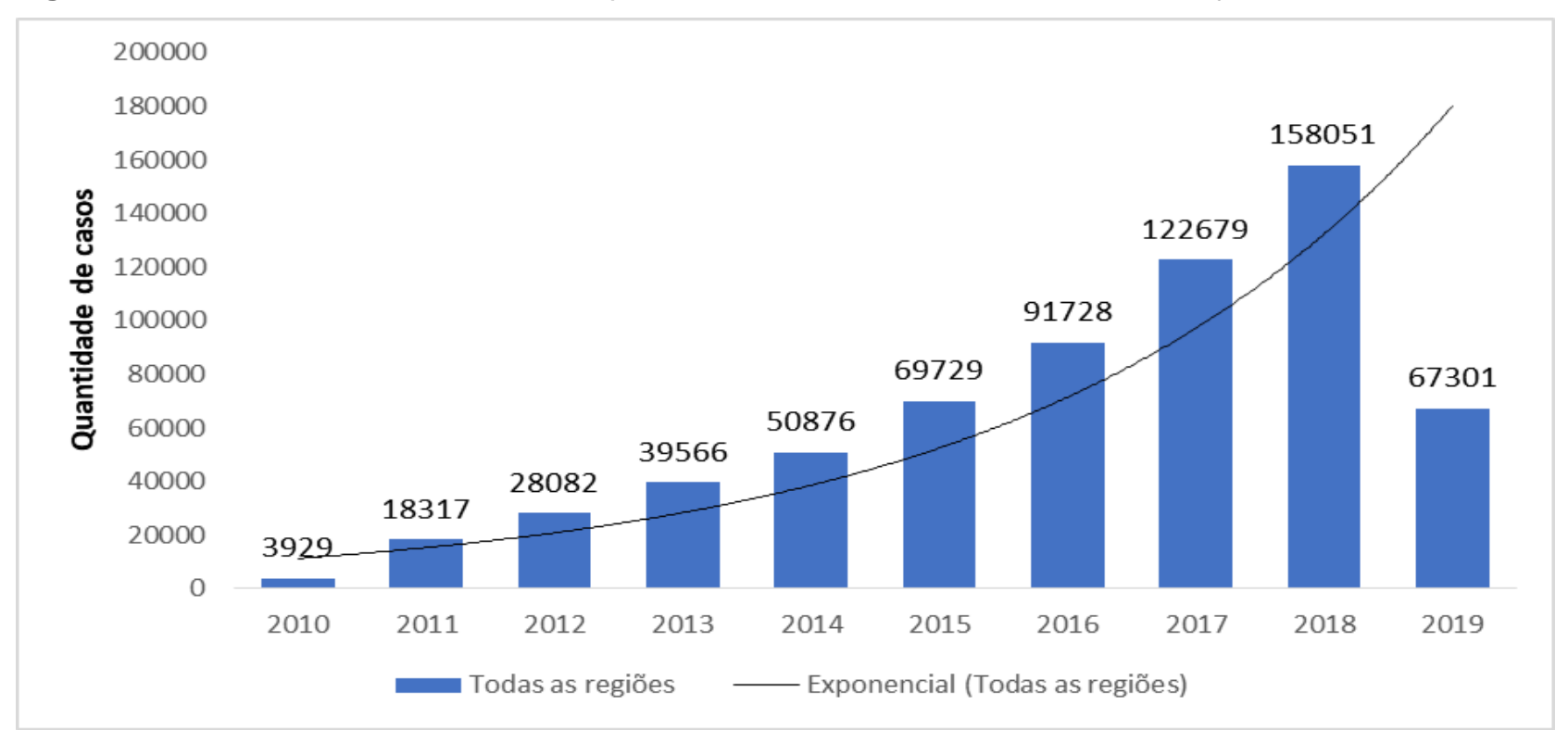

Fonte: Santos LG, et al., 2020.

A região Norte foi a que menos casos foram notificados. A taxa de detecção, por 100.000 habitantes, de Sífilis Adquirida, por ano, foi crescente, de 1,0\% ( $n=156)$ em 2010, 3,2\% ( $n=520)$ em 2011, 5,3\% ( $n=873)$ em 2012, 8,7\% ( $n=1.488)$ em 2013, 10,1\% ( $n=1.751)$ em 2014, 12,6\% ( $n=2.211)$ em 2015, 23,3\% ( $n=$ 4.139) em 2016, 34,1\% ( $n=6.124)$ em $2017,54,4 \%(n=9.890)$ em 2018 , e um total de 4.860 acometidos no primeiro semestre de 2019.

A taxa de detecção de Sífilis Adquirida, por 100.000 habitantes, na região Nordeste, por ano, foi de $0,7 \%$ $(n=360)$ em 2010; 3,9\% ( $n=2.061)$ em 2011; 4,7\% ( $n=2.535)$ em 2012; 6,1\% ( $n=3.414)$ em 2013; 7,8\% $(n=$ 4.377) em 2014; 12,2\% ( $n=6.878)$ em 2015; 18,5\% ( $n=10.549)$ em 2016; $27,4 \%(n=15.704)$ em 2017 ; 46,9\% ( $n=26.644)$ em 2018; e 11.196 casos totais no primeiro semestre de 2019 .

Já na região Sul, em 2010, a taxa foi de 1,2\% ( $n=315)$; em 2011, 5,7\% ( $n=1.583)$; em 2012, 14,3\% ( $n=$ 3.974); em 2013, 21,4\% ( $n=6.173)$; em 2014, 35,4\% ( $n=10.286)$; em 2015, 59,7\% $(n=17.458)$; em 2016, $74,1 \%(n=21.803)$; em 2017, 99,1\% ( $n=29.365)$; em 2018, teve 36.808 casos; e 2019, 16.239 casos.

A região Sudeste foi a que mais casos foram notificados. A taxa de detecção, por 100.000 habitantes, de Sífilis Adquirida, por ano, foi de 3,2\% ( $n=2.579)$ em 2010; $16,4(n=13.282)$ em $2011 ; 23,9 \%(n=19.461)$ em 2012; 31,6\% ( $n=26.723)$ em 2013; 38,0\% ( $n=32.342)$ em 2014; 46,0\% ( $n=39.451)$ em $2015 ; 57,6 \%(n=$ 49.700) em 2016; 73,0\% ( $n=63.490)$ em 2017; 81,9\% ( $n=71.842)$ em 2018; e em 2019 (até junho), um total de 29.339 casos.

Na região Centro-Oeste, a Sífilis Adquirida acometeu, por ano, por 100.000 habitantes, $3,7 \%(n=518)$ em 2010; 6,1\% $(n=870)$ em 2011; 8,6\% ( $n=1.235)$ em 2012; 11,8\% ( $n=1.764)$ em $2013 ; 13,9 \%(n=2.116)$ em 2014; 24,1\% ( $n=3.726)$ em 2015; 35,3\% $(n=5.532)$ em 2016; 50,3\% ( $n=7.985)$ em $2017 ; 79,9 \%(n=$ 12.855) em 2018; e no primeiro semestre de 2019, 5.656 casos.

De 2010 a junho de 2019, o Mato Grosso do Sul foi o estado com mais acometidos do Centro-Oeste, com $36,7 \%(n=15.524)$, e Mato Grosso foi o estado com menor número, com $13,5 \%(n=5.712)$. No Sudeste, São Paulo teve maiores números, com 61,9\% ( $n=215.563)$ dos casos e Espírito Santo, o menos, com 6,6\% $(n=22.987)$ dos casos. No Sul, o Rio Grande do Sul foi o estado com mais casos, 47,9\% ( $n=$ 69.036), e o Paraná com menos, $26,0 \%(n=37.481)$. O estado com mais casos no Nordeste foi a Bahia, $31,5 \%(n=26.395)$, e Alagoas teve menos casos, $2,14 \%(n=1.796)$. Já no Norte, o estado com maior índice de casos de Sífilis Adquirida, no mesmo intervalo de tempo, foi o Amazonas, 36,5\% ( $n=11.715$ ), e o menor, Amapá, 3,7\% ( $n=1.210)$ (Figura 2). 
Figura 2 - Comparativo entre os estados por região com maior e menor índice de casos de Sífilis Adquirida (2010 a junho de 2019).

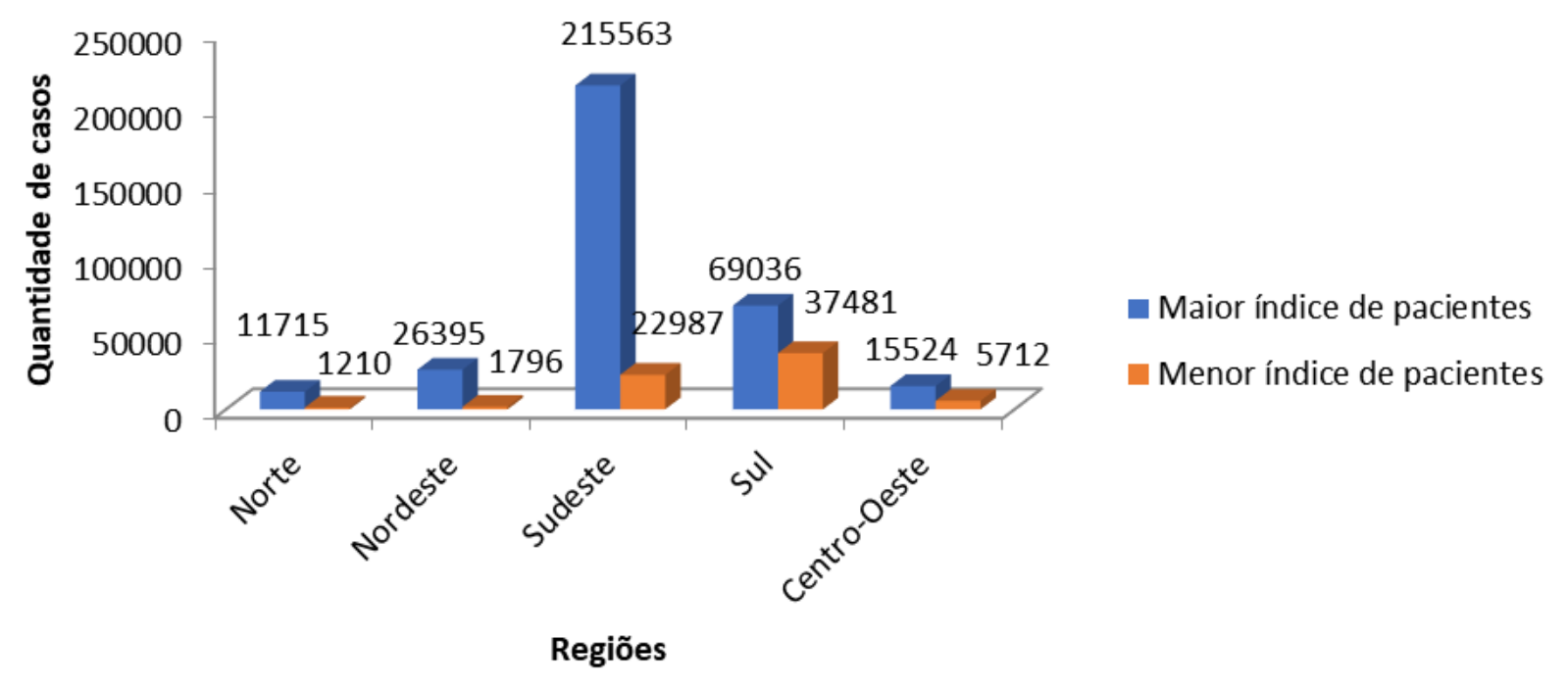

Fonte: Santos LG, et al., 2020.

Os casos totais notificados nos anos estudados do gênero masculino tiveram uma taxa de $59,6 \%$ ( $n=$ 387.312) e do feminino, 40,4\% ( $n=262.670)$ (Figura 3).

Figura 3 - Relação comparativa de número de casos de Sífilis Adquirida nos gêneros masculino e feminino (2010 a junho de 2019).

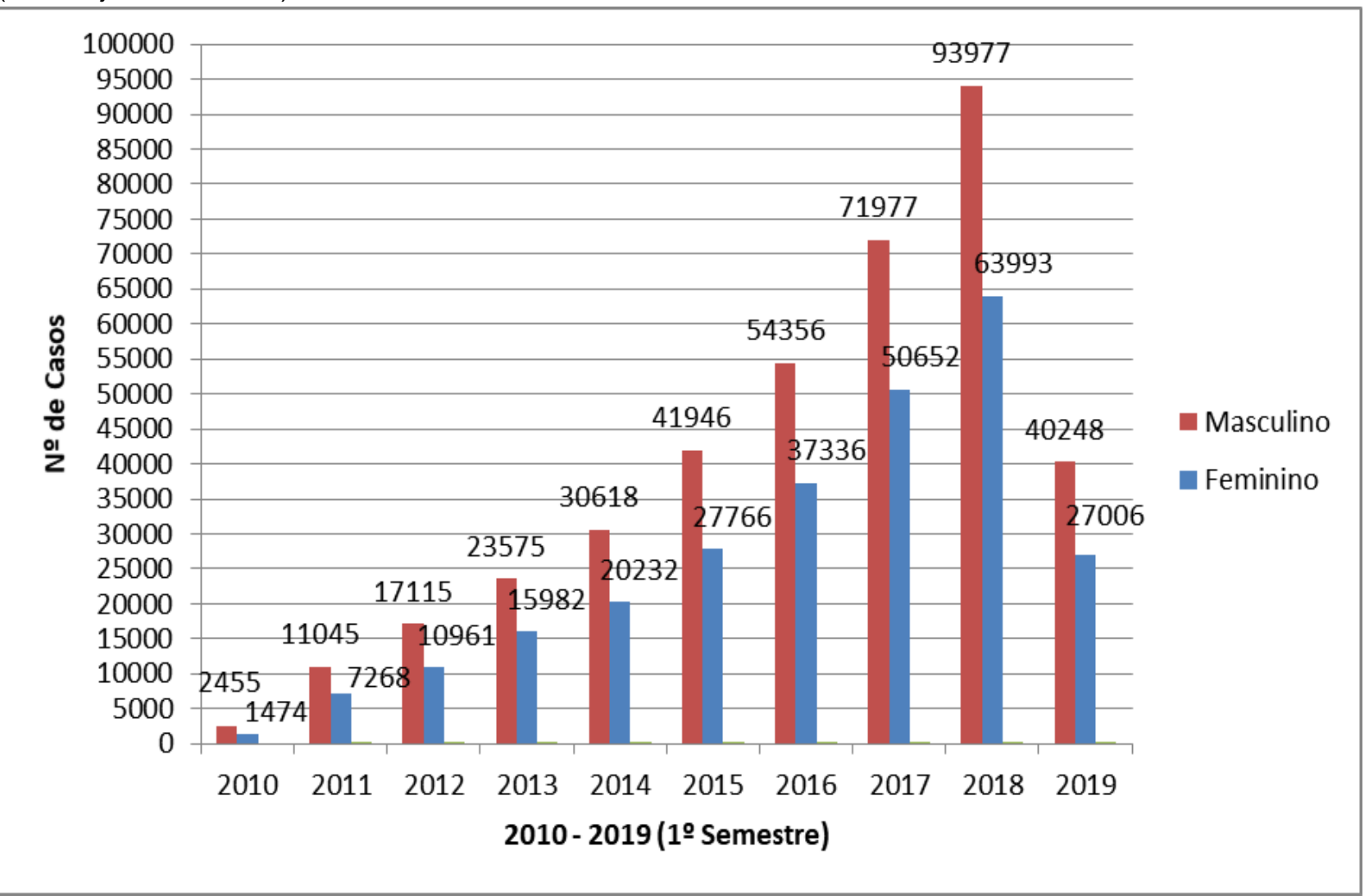

Fonte: Santos LG, et al., 2020. 
Observa-se que, em relação à faixa etária dos acometidos por Sífilis Adquirida no Brasil, o maior número de casos desde $o$ início das notificações da doença, em todos os anos, foi de 20 a 29 anos, com 33,6\% ( $n=$ 218.405). Já os menores de 13 anos tiveram as menores taxas, com o total de $0,7 \%(n=4.586)$. De 30 a 39 anos, houve uma taxa total de $22,0 \%(n=142.978)$; de 50 anos ou mais, $19,2 \%(n=124.546)$; de 40 a 49 anos, $14,3 \%(n=93.087)$; e os acometidos de 13 a 19 anos, $10,3 \%(n=66.656)$.

Quanto à escolaridade, a porcentagem total de pessoas com Sífilis Adquirida de 2010 a junho de 2019 foi de $1,2 \%(n=7.812)$ para analfabetos; $5,8 \%(n=37.413)$ para $1^{\text {a }}$ a $4^{\text {a }}$ incompleta; $3,6 \%(n=23.662), 4^{\text {a }}$ completa; $12,1 \%(n=78.404), 5^{\text {a }}$ a $8^{\text {a }}$ incompleta; $7,9 \%(n=51.108)$, ensino fundamental completo; $8,5 \%(n=$ 55.326), ensino médio incompleto; $17,0 \%(n=110.295)$, ensino médio completo; $3,1 \%(n=20.288)$, superior incompleto; e 3,8\% ( $n=24.649)$, superior completo (Tabela 1).

O total de pessoas com Sífilis Adquirida de cor branca teve taxa de $37,8 \%(n=245.827)$; de cor parda, $34,2 \%(n=222.566)$; preta, 9,7\% ( $n=62.819)$; amarela, $0,7 \%(n=4.747)$; indígena, $0,6 \%(n=3.636)$; e ignorado, $17,0 \%(n=110.663)$ (Tabela 1). 
Revista Eletrônica Acervo Científico / Electronic Journal Scientific Collection | ISSN 2595-7899

Tabela 1 - Distribuição da escolaridade e raça dos acometidos pela Sífilis Adquirida (2010 a junho de 2019).

\begin{tabular}{|c|c|c|c|c|c|c|c|c|c|c|}
\hline \multirow{2}{*}{$\begin{array}{l}\text { Característica } \\
\text { Escolaridade }\end{array}$} & \multicolumn{10}{|c|}{ Anos (N) } \\
\hline & 2010 & 2011 & 2012 & 2013 & 2014 & 2015 & 2016 & 2017 & 2018 & 2019 \\
\hline Analfabeto & 54 & 295 & 392 & 548 & 655 & 828 & 1079 & 1393 & 1867 & 701 \\
\hline $1^{\text {a }}$ a $4^{\underline{a}}$ incompleta & 216 & 1473 & 2181 & 2803 & 3336 & 4029 & 4893 & 6712 & 8480 & 3290 \\
\hline $4^{\mathrm{a}}$ completa & 166 & 1000 & 1392 & 1763 & 2126 & 2596 & 3197 & 4130 & 5241 & 2051 \\
\hline $5^{\mathrm{a}}$ a $8^{\mathrm{a}}$ incompleta & 427 & 2452 & 3728 & 5317 & 6854 & 8679 & 11348 & 14461 & 17827 & 7311 \\
\hline Ensino fundamental completo & 315 & 1738 & 2400 & 3372 & 4144 & 5618 & 6861 & 9419 & 12137 & 5104 \\
\hline Ensino médio incompleto & 184 & 1159 & 1870 & 2838 & 3922 & 5743 & 7872 & 10848 & 14556 & 6334 \\
\hline Ensino médio completo & 430 & 2542 & 4374 & 6190 & 8123 & 11355 & 15284 & 20801 & 28313 & 12883 \\
\hline Superior incompleto & 61 & 383 & 756 & 1099 & 1557 & 2228 & 2989 & 3984 & 4962 & 2269 \\
\hline Superior completo & 111 & 569 & 1059 & 1409 & 2019 & 2659 & 3360 & 4627 & 6052 & 2784 \\
\hline Não se aplica & 18 & 109 & 172 & 244 & 321 & 459 & 556 & 813 & 972 & 427 \\
\hline Ignorado & 1947 & 6597 & 9758 & 13983 & 17819 & 25535 & 34289 & 45491 & 57644 & 24147 \\
\hline Total & 3929 & 18317 & 28082 & 39566 & 50876 & 69729 & 91728 & 122679 & 158051 & 67301 \\
\hline \multicolumn{11}{|l|}{ Raça } \\
\hline Branca & 1200 & 7085 & 10858 & 15527 & 20190 & 27686 & 35094 & 46927 & 57226 & 24034 \\
\hline Preta & 338 & 1669 & 2614 & 3501 & 4656 & 6433 & 8589 & 11959 & 16308 & 6752 \\
\hline Amarela & 22 & 87 & 151 & 215 & 319 & 431 & 574 & 1006 & 1399 & 543 \\
\hline Parda & 999 & 5723 & 8628 & 12481 & 16353 & 21773 & 30566 & 42368 & 58181 & 25494 \\
\hline Indígena & 34 & 123 & 150 & 172 & 218 & 299 & 507 & 691 & 1111 & 331 \\
\hline Ignorado & 1336 & 3630 & 5681 & 7670 & 9140 & 13107 & 16398 & 19728 & 23826 & 10147 \\
\hline Total & 3929 & 18317 & 28082 & 39566 & 50876 & 69729 & 91728 & 122679 & 158051 & 67301 \\
\hline
\end{tabular}

Fonte: Santos LG, et al., 2020. 


\section{DISCUSSÃO}

A Sífilis é uma doença de notificação compulsória, sendo os casos confirmados notificados à Vigilância Epidemiológica, o que torna a medida imprescindível para interromper a cadeia de transmissão e indicar as medidas de controle (COELHO JMR, et al., 2018). No Brasil, as informações são registradas no Sistema de Informação de Agravos de Notificação (SINAN), computando dados de identificação como sexo, idade, raça/cor da pele e escolaridade (LUPPI CG, et al., 2018).

No presente estudo, notou-se crescente o número de casos de Sífilis Adquirida no Brasil no decorrer dos anos estudados, sendo observados 3.929 casos no ano de 2010, seguido de aumentos progressivos no decorrer dos anos, chegando a 67.301 casos apenas no primeiro semestre de 2019. Números esses que podem ser justificados pelo aumento da notificação dos casos no país e intensificação da vigilância pelas secretarias de saúde (ANDRADE HS, et al., 2019; DIAS APS, et al., 2018).

Esse grande aumento também pode ser respaldado na literatura quando Souza BSO, et al. (2018) afirmam que, apesar das facilidades de diagnóstico e do tratamento disponíveis na rede básica do Sistema Único de Saúde (SUS), as taxas alarmantes de Sífilis Adquirida em todo o país relacionam-se à diminuição das práticas seguras de sexo. As mudanças no comportamento sexual se deveram à redução na frequência do uso do preservativo masculino, favorecendo a vulnerabilidade às Infecções Sexualmente Transmissíveis (ISTs), assim como a tendência do abandono dos seus métodos de prevenção entre parceiros sexuais com o intuito de melhorar o desempenho e prazer durante a relação sexual (ANDRADE HS, et al., 2019; LUPPI CG, et al., 2018).

No início de 2014, a falta da matéria prima para a fabricação da Penicilina Benzatina, indicada para o tratamento de sífilis adquirida e em gestantes, ocasionou desabastecimento do medicamento em todo o país, ilustrando um aumento de $32,7 \%$ de casos em 2015. Além disso, a automedicação com antimicrobianos, um importante fator de risco para aumento da resistência bacteriana e falha no tratamento, também pode ter contribuído para o aumento da doença (COELHO JMR, et al., 2018; DIAS APS, et al., 2018; LIMA BCF, et al., 2018; SANTOS A, et al., 2017; SOUZA CC, et al., 2018).

Alguns fatores que podem contribuir para o aumento nos índices da doença foram relatados por Rodrigues ARM, et al. (2016), listando alto risco entre profissionais do sexo e usuários de drogas, devido a vulnerabilidade a ISTs e o compartilhamento de injetáveis. Nesse cenário, é valido ressaltar que a associação entre sífilis e HIV (Vírus da Imunodeficiência Humana) está bem documentada por diversos estudos tendo em vista que a elevada prevalência da sífilis entre portadores do HIV cresceu nas últimas décadas (SANTOS A, et al., 2017). A falta de informação sobre a doença também se mostrou importante devido a recusa ao tratamento como consequência da falta de conhecimento (RODRIGUES ARM, et al., 2016).

As regiões mais prevalentes de Sífilis Adquirida observadas nos anos de 2010 a junho de 2019 foram no Sudeste ( $n$ total $=348.209$ ) seguido da região Sul ( $n$ total $=144.004)$, Nordeste ( $n$ total=83.718), CentroOeste ( $n$ total $=42.257$ ), e Norte ( $n$ total=32.012), nessa ordem. Esses dados podem ser corroborados quando COELHO JMR et al. (2018) trazem em seu estudo que, no período de 2010 a junho de 2017, 59,2\% dos casos estavam concentrados na região Sudeste e 21,2\% no Sul. Em consenso, Luppi CG, et al. (2018), analisando o período de 2010 a 2016, em que foram notificados 227.663 casos de Sífilis Adquirida em adultos no país, referem que o estado de São Paulo foi responsável por $44 \%$ desse total.

A partir de 2012 é observado, no presente estudo, o aumento de casos notificados na região Sul, tornando-a a segunda mais prevalente desse ano até junho de 2019. Andrade HS, et al. (2019) afirmam que isso se deve à intensificação da vigilância pelas secretarias de saúde, o que pode ter aumentado a ocorrência de notificações no estado do Rio Grande do Sul. Apesar desse cenario, LEILA RS, et al. (2018) expõem que 0 aumento dos casos decorre das dificuldades das estratégias de tratamento e de conscientização da população quanto à forma de prevenção.

De 2010 a junho de 2019, em relação à raça, pessoas com Sífilis Adquirida de cor branca tiveram taxa de 37,8\%; parda, 34,2\%; ignorado, 17,0\%; preta, 9,7\%; amarela, 0,7\%; e indígena, $0,6 \%$ apenas. No caso 
dos povos indígenas do Brasil, a subnotificação pode estar associada ao preenchimento incompleto e incorreto das fichas de notificação e a não alimentação do Sistema pelas unidades notificadoras (TIAGO ZS, et al., 2017).

Em relação ao gênero, o masculino teve uma taxa de 59,6\% no intervalo de anos estudado, mostrando a predominância de casos confirmados nos homens, mesmo somando Sífilis Adquirida em mulheres com casos de Sífilis em gestantes (PEDER LD, et al., 2019; SOUZA CC, et al., 2018). Eles estão associados à maior exposição à multiplicidade de parceiros sexuais e ao baixo índice de uso do preservativo regular (PEDER LD, et al., 2019). Apesar disso, Soares SCL, et al. (2019) relatam que o risco associado à sífilis é superior para o sexo feminino, sendo de $78 \%$, devido à dificuldade de negociar o uso do preservativo com o parceiro. Entretanto, Maciel RB, et al. (2017) sugerem aumento significativo de mulheres portadoras de sífilis, tendo em vista que elas têm apresentado maior preocupação com a saúde nos últimos anos. Contribuindo para esse fato, a falta de médicos específicos para homens também corrobora para a falta de conhecimento e prevenção da doença, assim como o receio dos homens em procurar ajuda. Como também pode ser citado as Unidades básicas de saúde funcionam em horários comerciais dificultando o acesso dos homens e mulheres que trabalham.

A faixa etária de 20 a 29 anos foi a predominante, representando a maior parte dos registros em todos os anos (MACIEL RB, et al., 2017; SOUZA BSO, et al., 2018; SOARES SCL, et al., 2019). Ademais, essa faixa etária foi significativa em ambos os sexos (SILVA ZF, et al., 2017). Isso pode se dever ao fato de ser uma faixa de idade com maior desempenho da vida sexual, além de que o número expressivo de adolescentes com a infecção atesta o início precoce e desprotegido da vida sexual (ANDRADE HS, et al., 2019; SOUZA BSO, et al., 2018).

Quanto à escolaridade, as taxas de Sífilis Adquirida de 2010 a junho de 2019, foram de 1,2\% para analfabetos, 5,8\% para $1^{\underline{a}}$ a $4^{\underline{a}}$ incompleta; $3,6 \%$, $4^{\underline{a}}$ completa; $12,1 \%$, $5^{\underline{a}}$ a $8^{\underline{a}}$ incompleta; $7,9 \%$, ensino fundamental completo; $8,5 \%$, ensino médio incompleto; $17,0 \%$, ensino médio completo; $3,1 \%$, superior incompleto; e 3,8\%, superior completo. Dessa forma, faz-se necessário destacar que há maior número de pacientes com Sífilis Adquirida nos casos em que a população não finalizou a educação básica.

A informação pode ser vista como um marcador importante, tendo em vista a relação direta da baixa escolaridade ao limitado conhecimento sobre as medidas de prevenção das ISTs, sendo fundamental o conhecimento da doença e dos métodos de prevenção que incluem o sexo seguro (SOUZA BSO, et al., 2018; PEDER LD, et al., 2019; RODRIGUES ARM, et al., 2016). As falhas no sistema de saúde com deficiência no processo educacional da população também são apontadas por Rodrigues ARM, et al. (2016) como um grave problema de saúde pública, dificultando, assim, o processo de disseminação da informação.

\section{CONCLUSÃO}

Apesar de a Sífilis Adquirida ser uma doença com diagnóstico e tratamento bem definidos, são nitidamente notados, no estudo, os aumentos em sua incidência no Brasil. Fatores como início precoce da vida sexual, relações sexuais desprotegidas e intensificação das notificações podem estar relacionados com esse aumento. Assim, é válido que haja ações de entidades governamentais e profissionais da saúde para a prevenção e controle da sífilis, estimulando-se à adesão ao tratamento e ao seu seguimento, ao uso de preservativo e à procura dos serviços de saúde para prevenção de diagnóstico precoce.

\section{REFERÊNCIAS}

1. ANDRADE HS, et al. Caracterização epidemiológica dos casos de sífilis em mulheres. Ciência \& Saúde, 2019; 12(1), 32124

2. BRASIL. Ministério da Saúde. Secretaria de Vigilância em Saúde. Departamento de DST, Aids e Hepatites Virais. Protocolo Clínico e Diretrizes Terapêuticas para Atenção Integral às Pessoas com Infecções Sexualmente Transmissíveis. Brasília, 2019; $1^{a}$ ed.; 55p. 
3. BRASIL. Ministério da Saúde. Secretaria de Vigilância em Saúde. Departamento de Vigilância, Prevenção e Controle das Infecções Sexualmente Transmissíveis, do HIV/Aids e das Hepatites Virais. Agenda de Ações Estratégicas para Redução da Sífilis no Brasil/Ministério da Saúde, Secretaria de Vigilância em Saúde, Departamento de Vigilância, Prevenção e Controle das Infecções Sexualmente Transmissíveis, do HIV/Aids e das Hepatites Virais. Brasília, 2017; 15p.

4. BRASIL. Ministério da Saúde. Secretaria de Vigilância em Saúde. Boletim Epidemiológico Sífilis. Brasília, 2019; $16 p$.

5. COELHO JMR, et al. Sífilis: um panorama epidemiológico do Brasil e do município de Volta Redonda/RJ. Brazilian Journal of Health Review, 2018; 1(1), 128-147.

6. DIAS APS, et al. A sífilis no atual cenário brasileiro. Health Research Journal, 2018; 1(2), 1-21.

7. FOCACCIA R, VERONESI R. Tratado de infectologia. 5a ed. São Paulo: Editora Atheneu, 2005; 1543-1550p.

8. KASPER DL, FAUCI AS. Doenças infecciosas de Harrison. 2ª ed. Porto Alegre: AMGH, 2015; 579-581p.

9. LEILA RS, et al. Conhecimento de mulheres com diagnóstico de sífilis e suas escolhas terapêuticas. Cuba Salud, 2018.

10. LIMA BCF, et al. Incidência de Sífilis no município de Almirante Tamandaré nos anos de 2010-2018. Revista UNIANDRADE, 2018; 19(3), 133-138.

11. LUPPI CG, et al. Fatores associados à coinfecção por HIV em casos de sífilis adquirida notificados em um Centro de Referência de Doenças Sexualmente Transmissíveis e Aids no município de São Paulo, 2014. Epidemiologia e Serviços de Saúde, 2018; 27, 1-12.

12. MACIEL RB, et al. Perfil epidemiológico dos casos de sífilis na cidade de Americana (SP) de 2005 a 2015 . Revista de Epidemiologia e Controle de Infecção, 2017; 7(3), 161-168.

13. PEDER LD, et al. Aspectos Epidemiológicos da Sífilis no Sul do Brasil: cinco anos de experiência. Revista EVSRevista de Ciências Ambientais e Saúde, 2019; 46(1).

14. RODRIGUES ARM, et al. Atuação de enfermeiros no acompanhamento da Sífilis na Atenção Primária. Journal of Nursing UFPE, 2016; 10(4):1247-1255.

15. SANTOS A, et al. Um novo desafio para a saúde pública: sífilis. Diversitates International Journal, 2017; 9(2), 65-81.

16. SILVA ZF, et al. Pacientes portadores de sífilis atendidos em uma unidade terciária em Fortaleza: perfil sociodemográfico. Revista Brasileira de Análises Clínicas, 2017; 49(1), 105-109.

17. SOARES SCL, et al. Sífilis em privados de liberdade em uma unidade prisional no interior de Rondônia. Brazilian Journal of Health Review, 2019; 2(2), 2195-2205.

18. SOUZA BSO, et al. Análise epidemiológica de casos notificados de sífilis. Revista da Sociedade Brasileira de Clínica Médica, 2018; 16(2), 94-98.

19. SOUZA CC, et al. Perfil epidemiológico das pessoas residentes em Criciúma com diagnóstico de Sífilis Adquirida no período de 2012 a 2016. Revista Interdisciplinar de Estudos em Saúde, 2018; 7(1), 263-276.

20. TIAGO ZS, et al. Subnotificação de sífilis em gestantes, congênita e adquirida entre povos indígenas em Mato Grosso do Sul, 2011-2014. Epidemiologia e Serviços de Saúde, 2017; 26, 503-512. 